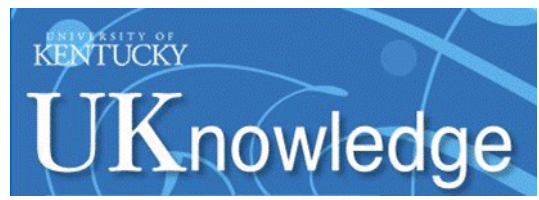

University of Kentucky

UKnowledge

$10-2019$

\title{
Systematic Comparison of Two Axial Flux PM Machine Topologies: Yokeless and Segmented Armature versus Single Sided
}

\author{
Narges Taran \\ University of Kentucky, narges.taran@uky.edu \\ Greg Heins \\ Regal Beloit Corporation, Australia \\ Vandana Rallabandi \\ University of Kentucky, vandana.rallabandi@uky.edu \\ Dean Patterson \\ Regal Beloit Corporation, Australia
}

Dan M. Ionel

University of Kentucky, dan.ionel@uky.edu

Follow this and additional works at: https://uknowledge.uky.edu/peik_facpub

Part of the Power and Energy Commons

Right click to open a feedback form in a new tab to let us know how this document benefits you.

\section{Repository Citation}

Taran, Narges; Heins, Greg; Rallabandi, Vandana; Patterson, Dean; and Ionel, Dan M., "Systematic Comparison of Two Axial Flux PM Machine Topologies: Yokeless and Segmented Armature versus Single Sided" (2019). Power and Energy Institute of Kentucky Faculty Publications. 11.

https://uknowledge.uky.edu/peik_facpub/11

This Conference Proceeding is brought to you for free and open access by the Power and Energy Institute of Kentucky at UKnowledge. It has been accepted for inclusion in Power and Energy Institute of Kentucky Faculty Publications by an authorized administrator of UKnowledge. For more information, please contact UKnowledge@lsv.uky.edu. 


\title{
Systematic Comparison of Two Axial Flux PM Machine Topologies: Yokeless and Segmented Armature versus Single Sided
}

\author{
Digital Object Identifier (DOI) \\ https://doi.org/10.1109/ECCE.2019.8913104
}

\section{Notes/Citation Information}

Published in 2019 IEEE Energy Conversion Congress and Exposition (ECCE).

(C) 2019 IEEE Copyright Notice. "Personal use of this material is permitted. Permission from IEEE must be obtained for all other uses, in any current or future media, including reprinting/republishing this material for advertising or promotional purposes, creating new collective works, for resale or redistribution to servers or lists, or reuse of any copyrighted component of this work in other works."

The document available for download is the authors' manuscript version that is accepted for publication. The final published version is copyrighted by IEEE and will be available as: N. Taran, G. Heins, V. Rallabandi, D. Patterson, and D.M. Ionel, "Systematic Comparison of Two Axial Flux PM Machine Topologies: Yokeless and Segmented Armature versus Single Sided," 2019 IEEE Energy Conversion Congress and Expo (ECCE), Baltimore, MD, 2019, pp. 1-6. 


\title{
Systematic Comparison of Two Axial Flux PM Machine Topologies: Yokeless and Segmented Armature versus Single Sided
}

\author{
Narges Taran ${ }^{1}$, Greg Heins ${ }^{2}$, Vandana Rallabandi ${ }^{1 *}$, Dean Patterson ${ }^{2}$, and Dan M. Ionel ${ }^{1}$ \\ ${ }^{1}$ SPARK Lab, ECE Department, University of Kentucky, Lexington, KY, USA \\ narges.taran@uky.edu, vandana.rallabandi@ieee.org, dan.ionel@ieee.org \\ ${ }^{2}$ Regal Beloit Corp., Research and Development, Rowville, VIC, Australia \\ greg.heins@ regalbeloit.com, dean.patterson@regalbeloit.com
}

\begin{abstract}
This paper systematically compares two axial flux permanent magnet (AFPM) machines designed for a university student racing car application: a double-rotor single-stator yokeless and segmented armature (YASA) structure, and a singlestator single-rotor configuration. Both machines are optimized for minimum loss and active weight using 3D finite element analysis and the highest performing candidate designs are compared in more detail. The studies indicate that the benefits offered by the YASA configuration over the single-stator single-rotor machine are achieved only for specific designs that are heavier. For the design space with lower mass, albeit with increased losses, the Pareto front designs overlap which shows the performance of the two machines is very close to each other.

Index Terms-Axial flux permanent magnet, yokeless and segmented armature, YASA, single sided, topology advantages, multi-objective optimization, 3D FEA.
\end{abstract}

\section{INTRODUCTION}

The disc shape of axial flux permanent magnet (AFPM) machines have opened up many configuration possibilities including yokeless and segmented armature (YASA). The YASA machine can be regarded as a next generation Torus type AFPM machine [1], [2] that combines winding arrangments of the NN and NS type Torus machines. The YASA structure has been proposed for traction application [3] and gained attention due to its high torque density and the segmented stator teeth structure that facilitates higher slot fill factor. On the other hand, the segmented structure adds to mechanical challenges [4], [5].

Previous studies have compared the performance of the YASA machine with other axial and radial flux machines [6][8]. However, optimal designs have not been considered, which may make the outcomes of such comparisons debatable. In this regard, this paper presents a systematic comparison for an example traction application of the two machine structures shown in Fig. 1. Optimization studies for both machines are performed to simultaneously achieve the objectives of minimum active material mass and minimum electromagnetic

* Dr. Vandana Rallabandi was with the SPARK Laboratory, ECE Department, University of Kentucky, Lexington, KY and is now with GE Research, Niskayuna, NY. loss, including the stator core loss and the dc copper loss. Due to the 3D flux path of the AFPM machines, 3D finite element analysis (FEA) is required for accurate performance estimation. Therefore, a surrogate assisted optimization process is employed that is capable of utilizing 3D FEA models for design evaluation. A comparative study is conducted for the optimum designs located on the Pareto front.

The next section describes the optimization method employed in this paper. Section III illustrates the AFPM machine topologies and their specifications for the optimal design. Section IV compares the obtained Pareto fronts and selected representative designs. The last section of the paper is devoted to concluding discussions.

\section{Kriging Surrogate Model Assisted OPTIMIZATION}

Three-dimensional FEA models provide the basis for an accurate design evaluation methodology, particularly for a machine with 3D magnetic flux or leakage flux path. In order to utilize time consuming 3D FEA models in the optimization process, a surrogate assisted algorithm is utilized, such as the one proposed in [9]. This is a two-level surrogate-assisted algorithm taking advantage of differential evolution and kriging models. The kriging models can be defined as interpolations of sampled data points that are composed of two elements; trend and residual component. The trend component can be a polynomial regression model while the residual component reduces the estimation errors by increasing the weight of closer neighbor samples. This can be formulated as

$$
\hat{Y}=\hat{X} \beta+r^{T} R^{-1}(Y-X \beta),
$$

where $\hat{X}$ is the design vector to be evaluated; $\hat{Y}$, response to be predicted based on the known sampled data points, i.e. $X$ and $Y . \beta$ is the matrix of regression coefficients that can be obtained using methods such as least squares. Kriging weights, $r^{T}$ and $R^{-1}$ are derived from the covariance function or semivariogram and maximum likelihood estimation (MLE). The covariance function can be defined by various kernel functions, in this study Matern covariance function is employed [10]. 


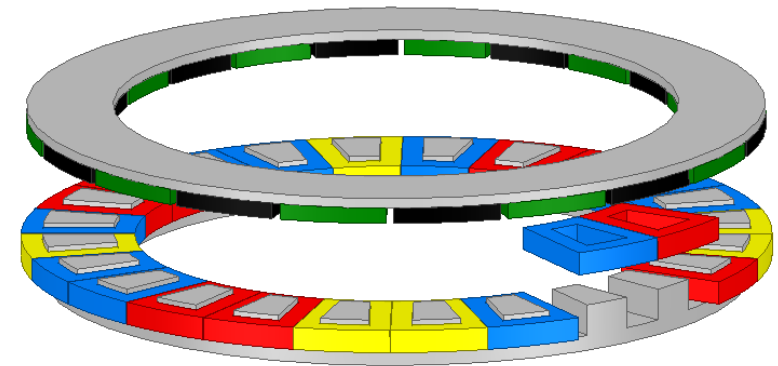

(a)

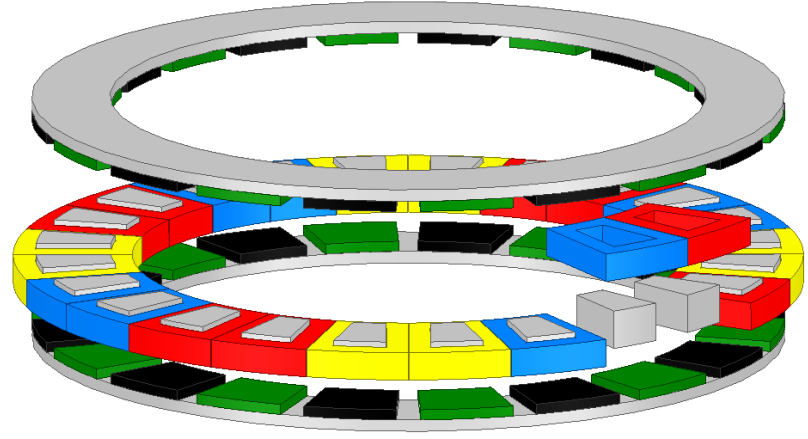

(b)

Fig. 1: The 3D parametric models of the two AFPM machines under study: (a) single-stator single-rotor, and (b) YASA with two rotors.

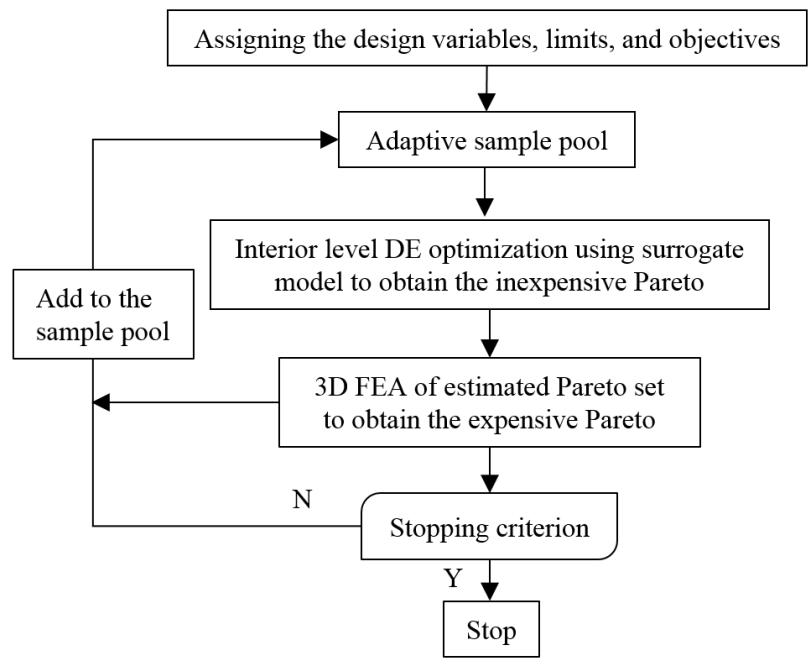

Fig. 2: The flowchart of the surrogate assisted optimization algorithm employing 3D FEA models.

This optimization flowchart, represented in Fig. 2, is based on a two level layout that provides an approach to evaluate only the most promising designs with computationally expensive 3D FEAs in the exterior loop, while the interior loop provides an approach for evaluating thousands of designs using inexpensive kriging surrogate interpolations. Considering that this approach drastically reduces the required number of FEA evaluations, it facilitates the application of 3D FEA. The algorithm is discussed in more detail in [9].
TABLE I: Independent optimization variables and their corresponding limits.

\begin{tabular}{llrl}
\hline Variable & Description & Min & Max \\
\hline$L_{a x}$ & Total axial length $[\mathrm{mm}]$ & 25.0 & 40.0 \\
$k_{r y}$ & rotor yoke ratio $=\frac{L_{r y}}{L_{a x}}$ & 0.1 & 0.16 \\
$k_{s y}$ & stator yoke ratio $=\frac{L_{s y}}{L_{a x}}$ & 0.13 & 0.20 \\
$k_{p m}$ & magnet length ratio $=\frac{L_{p m}}{L_{a x}}$ & 0.18 & 0.24 \\
$k_{d s}$ & split ratio $=\frac{I D_{s}}{O D_{s}}$ & 0.58 & 0.86 \\
$k_{o h}$ & over hang ratio $=\frac{\left(O D_{r}-O D_{s}\right)}{\left(O D-O D_{s}\right)}$ & -1.00 & 1.00 \\
$k_{s w}$ & slot width to slot pitch ratio $=\frac{w_{s}}{\tau_{s, i d}}$ & 0.58 & 0.88 \\
$k_{p}$ & pole arc to pole pitch ratio $=\frac{\tau_{p a}}{\tau_{p p}}$ & 0.64 & 0.96 \\
\hline
\end{tabular}

\section{Design Topologies And Setup}

The study reported here includes two AFPM machines, namely, a surface mounted (SPM) single sided 1-stator 1-rotor machine, shown in Fig. 1a, and a YASA machine configuration, represented in Fig. 1b. The motors are optimally designed for application in formula student design competition cars by the society of automotive engineers (SAE). Both machines have ratings of $70 \mathrm{Nm}$ at $6500 \mathrm{rpm}$. The reference single sided AFPM machine has been manufactured and presented in Fig. 3. The test results as shown in Fig. 4 present a good agreement between the 3D FEA and measurements, which attests to the accuracy of calculations.

In the optimization study, the current density is varied from design to design such that all produce the rated torque. Both machines incorporate 24 slots and 20 poles, concentrated winding and surface mounted magnets. The use of open slots and the application of bobbin wound coils result into the same high fill factor for the single sided machine as the YASA.

The motors are optimized employing accurate 3D FEA models for design evaluations. The objectives are to minimize active material mass and the electromagnetic loss, including the stator core loss and the dc copper loss. The winding and PM eddy current losses are estimated for the selected optimum designs.

The optimization process takes 8 variables for the single sided topology and 7 for the YASA, which features one less due to the absence of the stator yoke. The independent optimization variables are listed in Table I.

The diameter being the most influential design variable, the optimization is conducted for two constraint diameters. The outer diameter is fixed at $200 \mathrm{~mm}$ and $300 \mathrm{~mm}$ for two sets of studies. This incorporates the effect of the motor's physical dimensions on the best choice. The non-dominated or Pareto front optimum designs are obtained and plotted (Fig. 5). Elaborated comparative discussions in the following sections are based on the optimal designs on the Pareto front.

\section{Comparative Study}

\section{A. Pareto front designs}

The optimum designs from Fig. 5 produce the same rated torque, therefore a design with higher active material mass has lower specific torque $(\mathrm{Nm} / \mathrm{kg})$. It can be observed that for the designs with electromagnetic efficiency greater than 


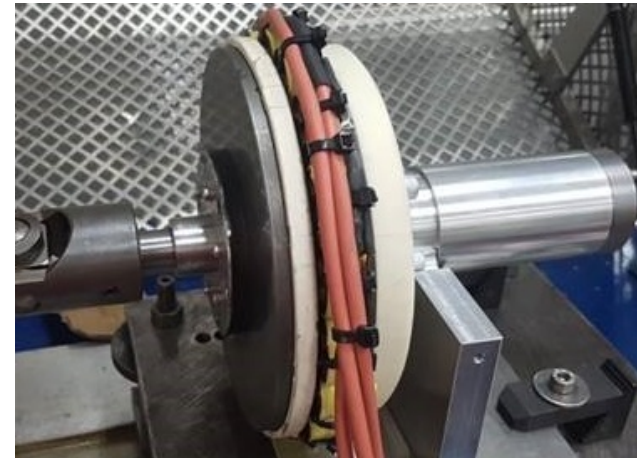

(a)

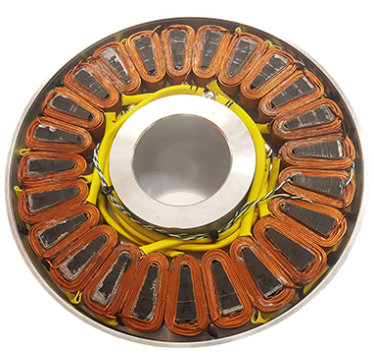

(b)

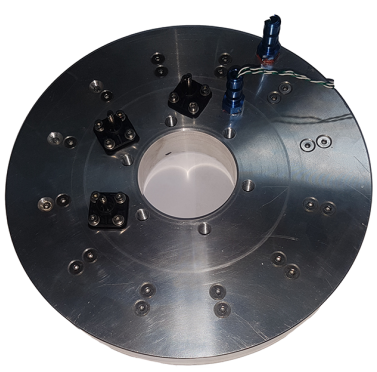

(c)
Fig. 3: The reference AFPM motor employed for the base design and experimental validation: (a) the test set-up, (b) the stator, (c) the oil cooling jacket.

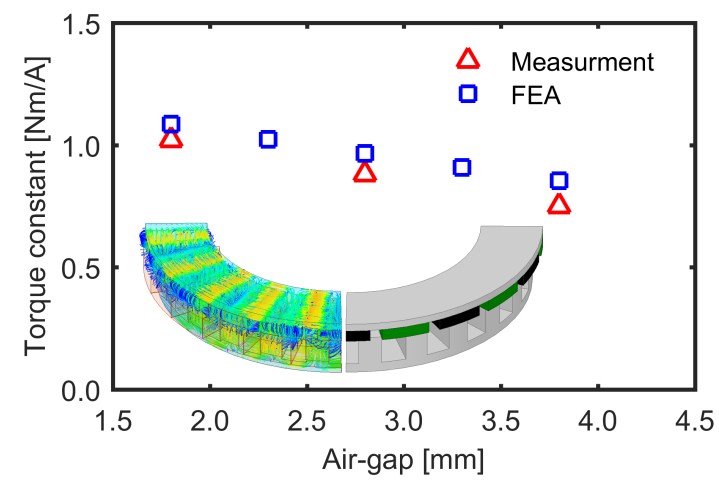

Fig. 4: The experimental validation of the FEA simulations.

about $98 \%$, the YASA topology has a higher specific torque compared to the single sided one. On the other hand, this high efficiency zone of the design space includes heavier machines. In applications where the mass is a vital concern, the right side of the plots in Fig. 5 would be more of interest. In this case, the single sided machine has slightly higher specific torque. Based on these results, it may be noted that generally claiming higher specific torque for the YASA topology does not hold true, as it is the case only for very low loss designs.

For both the envelope dimensions studied, i.e. OD of 200 $\mathrm{mm}$ and $300 \mathrm{~mm}$, the comparative performance represents a similar trend: for a mass sensitive application, the single sided topology may be at an advantage. On the other hand, if very efficient designs are of interest, higher specific torque can be gained by employing the YASA topology. The machines

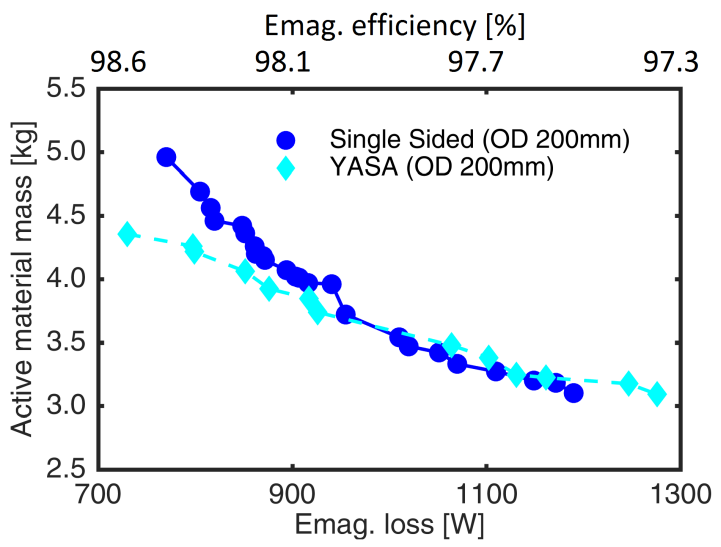

(a)

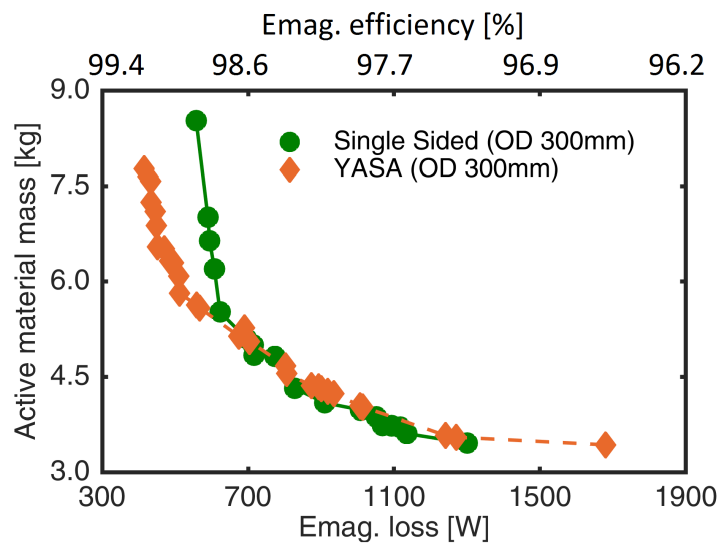

(b)

Fig. 5: The Pareto fronts for the topologies optimally designed for different envelopes: (a) outer diameter of $200 \mathrm{~mm}$, (b) outer diameter of $300 \mathrm{~mm}$

designed at a larger diameter constraint, can achieve even larger efficiency, albeit at the cost of increased mass.

The detailed distribution of optimization variables for the designs on the Pareto front is provided in Fig. 6. Some of the observations specific to this study are that the optimally designed YASA compared to single sided machines tend toward larger split ratios and slot widths. This may be explained by considering that the YASA topology has a lower stator core loss, due to the absence of the yoke, and hence higher flux density in the stator may be permissible, and thus, the machine can afford thinner teeth and therefore larger slot widths and split ratios.

In order to derive more general design guidelines and establish the limitations of the two topologies, the geometrical variables of the obtained Pareto designs are carefully investigated. For instance, the slot width and depth of the YASA machines were found to be larger than for the single sided ones, as shown in Fig. 7. A larger slot depth in the case of the single sided machine may not be beneficial as this also increases the leakage, more significantly than in the YASA machine. Considering the slope of the trend lines in Fig. 7, it can be inferred that lower loss and higher mass designs on the Pareto front generally have deeper slots and reduced slot 


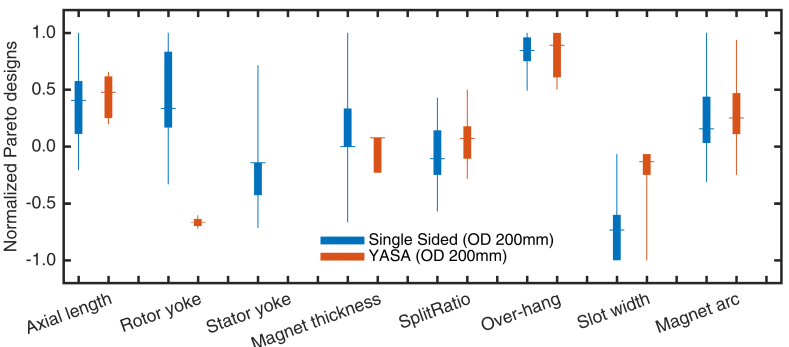

(a)

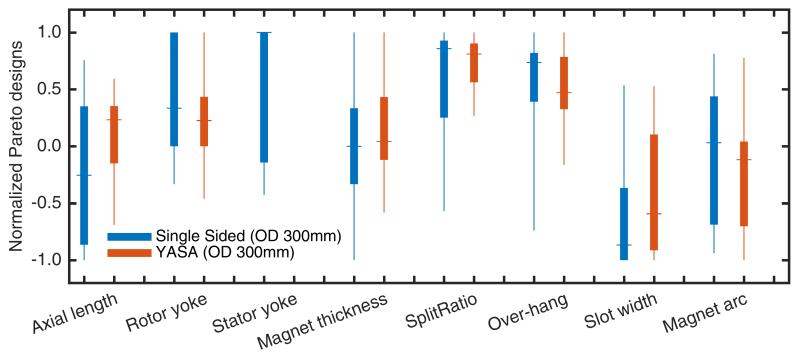

(b)

Fig. 6: The distribution of variables for optimum designs with total outer diameter of (a) $200 \mathrm{~mm}$, (b) $300 \mathrm{~mm}$.

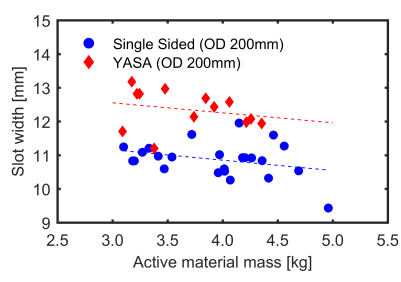

(a)

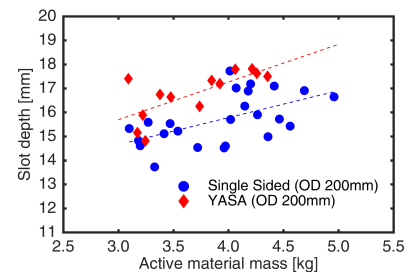

(b)
Fig. 7: The slot width and depth variation of Pareto front designs of (a) the single sided and (b) the YASA machine.

width.

The performance of the evaluated designs in the optimization are later investigated in three most frequent operating points. These points include the rated condition $(70 \mathrm{Nm}$ at $6500 \mathrm{rpm}$ ) which the optimization was performed at, as well as $14 \mathrm{Nm}$ and $35 \mathrm{Nm}$. Then each objective function for each design is calculated based on a weighted sum of that objective value in the three operating points. The weights are assigned according to an estimated operation duration. It was observed that the selected designs remain very close to the Pareto front.

\section{B. Mass components breakdown}

The breakdown of mass components for designs on the Pareto front is shown in Fig. 8. The copper mass required for optimum designs of the YASA machine is larger than for the single sided machine throughout the whole Pareto front. The magnet mass required for the optimum YASA design is also larger, except in designs with low total losses. The stator core mass of the YASA is smaller due to the elimination of the yoke, on the other hand it has higher rotor mass due to including two rotors.

\section{Loss components breakdown}

The breakdown of loss components for designs on the Pareto front is presented in Fig. 9. It is observed that the copper loss is dominant in the case of both topologies with the ratings and envelope size under study. The copper loss of the optimally designed YASA machines is higher than that of the single sided ones, except for the very heavy and high efficiency designs. The stator core loss of the single sided topology is larger than the YASA for all the designs on the Pareto front.

Two machines with similar mass and efficiency are selected in order to compare their PM and winding eddy current losses, and also obtain their efficiency map. Eddy current losses are not considered in the optimization in order to accelerate the 3D design evaluation process. These losses are assessed for the two selected optimal designs as follows.

The topology under study employs sintered Neodymium magnets. Magnet eddy current loss calculation with 3D timetransient FEA for the selected optimally designed YASA machine is shown in Fig. 10a. Should only one magnet per pole be used in the rotor, the eddy-current losses would be extremely high at $1 \mathrm{~kW}$ for the selected single sided topology and about twice that for the selected YASA topology. In the practical design each pole is segmented in 8 magnet pieces, resulting in a drastic reduction of losses.

The reasons for larger PM loss for the YASA machine topology include, thinner magnets that reduce the permeance coefficient and also doubled magnet surface area facing the air-gap that is more exposed to the flux density harmonics. These show that for this design problem, magnet segmentation or other magnet loss reduction methods need to be taken into account, particularly for the YASA machine.

The winding eddy current losses for the two selected optimum designs at rated operating conditions are calculated using 2D FEA. The calculations are conducted for scenarios with different numbers of turns, all with AWG 12. The current density is readjusted for the rated torque. Example FEA results for the selected YASA machine is presented in Fig. 10b.

The winding eddy current losses for the two machines are very close to each other except for the case with highest number of turns where the magnet passing eddy current losses are significant. This is mainly due to the larger slot depth for the YASA machine specified by the optimization algorithm. With identical conductors and number of turns, for the YASA machine with larger slot depth the conductors are located further away from the magnets, reducing flux density variation in conductors and hence mitigating the losses. It is expected that with identical slot depth, the YASA machine has larger winding eddy current losses as it features two air-gaps.

The efficiency maps for the selected optimum designs are calculated with 3D FEA and shown in Fig. 11. High efficiency performance is consistent at wider speed range as well. In order to simplify the comparison of the two efficiency maps a third plot is obtained by subtracting them, presented in Fig. $11 \mathrm{c}$. The positive values of this plot indicates higher efficiency of the single sided machine while the negative values show a higher efficiency for the YASA topology. 


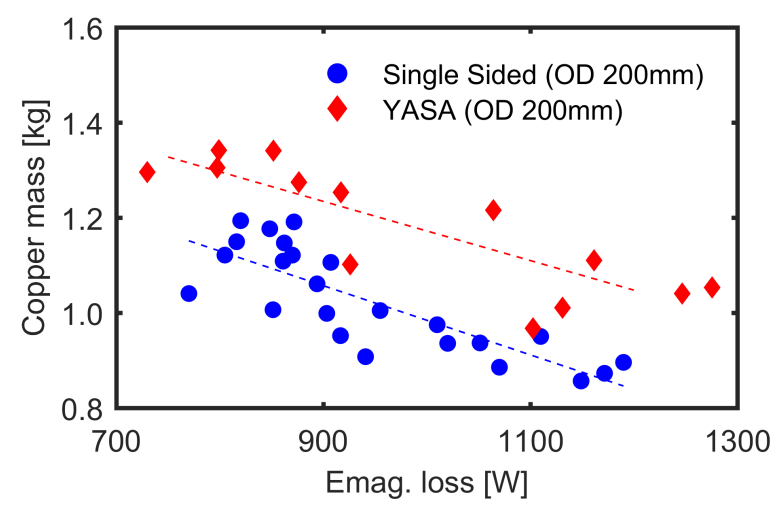

(a)

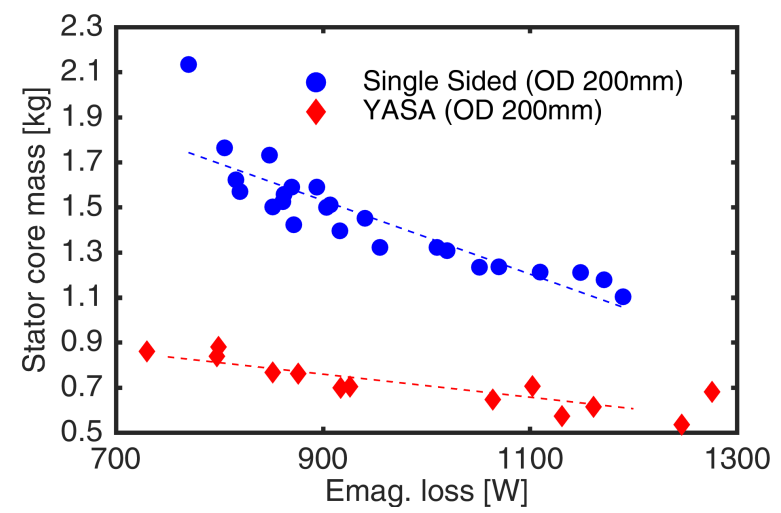

(c)

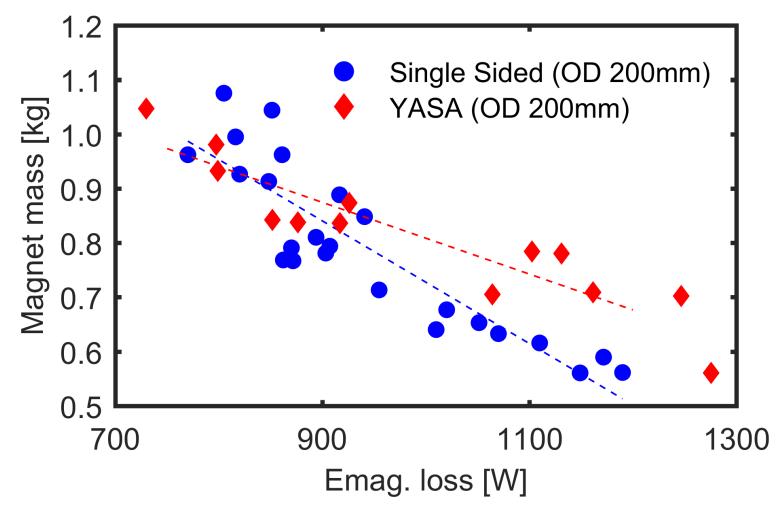

(b)

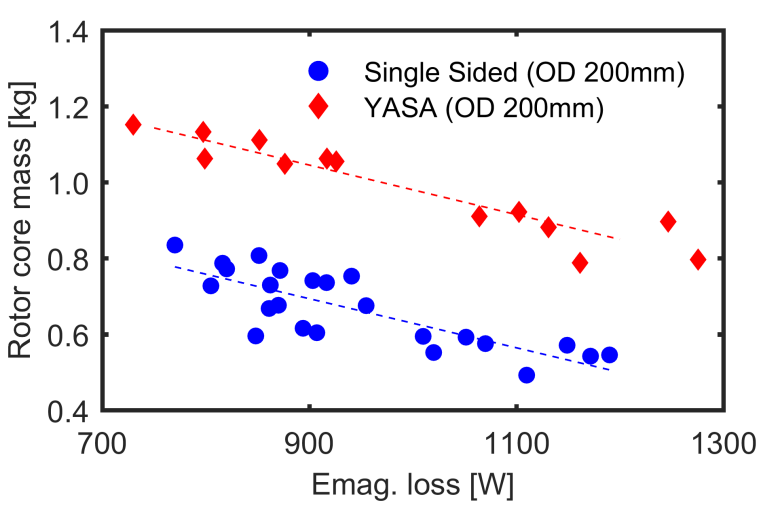

(d)

Fig. 8: The breakdown of mass components for the Pareto front designs of the topologies studied for an outer diameter of 200 mm. Similar trends were observed for the machines designed for the larger envelope with an outer diameter of $300 \mathrm{~mm}$.

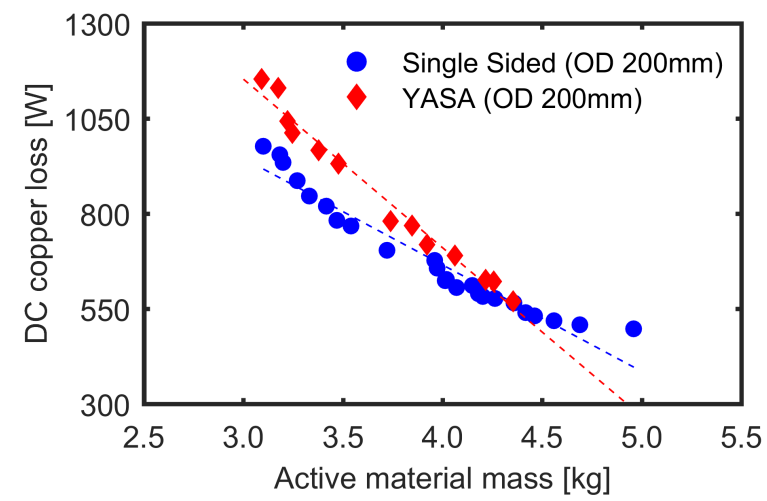

(a)

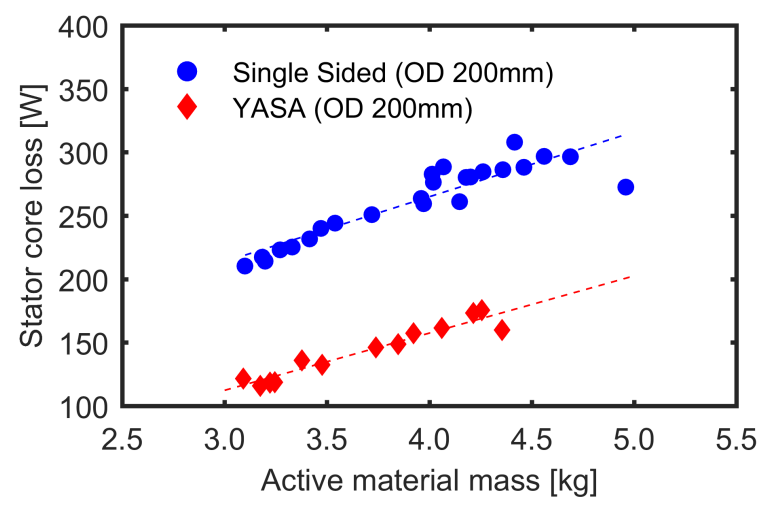

(b)

Fig. 9: The breakdown of loss components for Pareto front designs of the topologies studied for an outer diameter of 200 mm. Similar trends were observed for the machines designed for the larger envelope with an outer diameter of $300 \mathrm{~mm}$.

It can be seen that for the two selected designs, assuming identical cooling methods, the single-stator single-rotor machine performs favorably at higher torque and lower speed operating points where the copper loss is more significant. Therefore, it may perform better for driving cycles with more torque requirement while the YASA machine is better for traction motors with higher speed and lower torque requirements.

\section{CONClusion}

This paper comparatively studies the performance of a YASA topology as opposed to a single-rotor single-stator AFPM machine for a formula student race car. Both machine types have been optimized to achieve minimum mass and electromagnetic loss within a given dimensional envelope.

For identical heat transfer capabilities, reflected in the same 


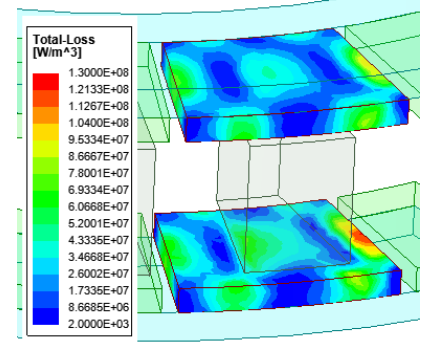

(a)

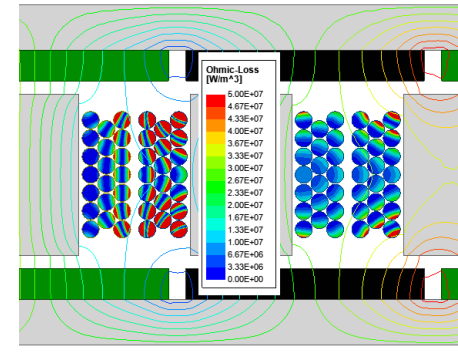

(b)
Fig. 10: Distribution of eddy current losses in the magnets (a) and the windings (b) of the selected YASA optimal designs.

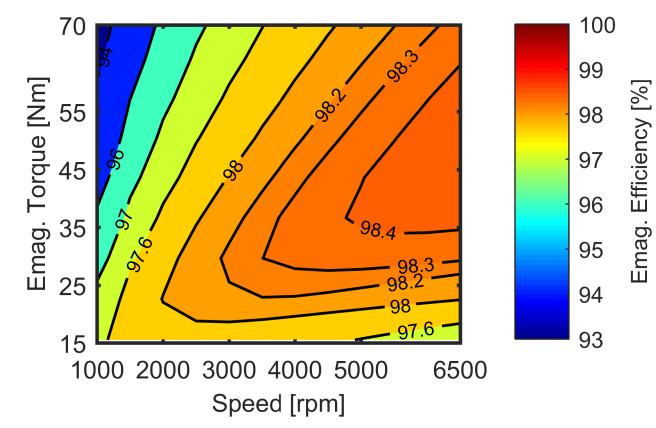

(a)

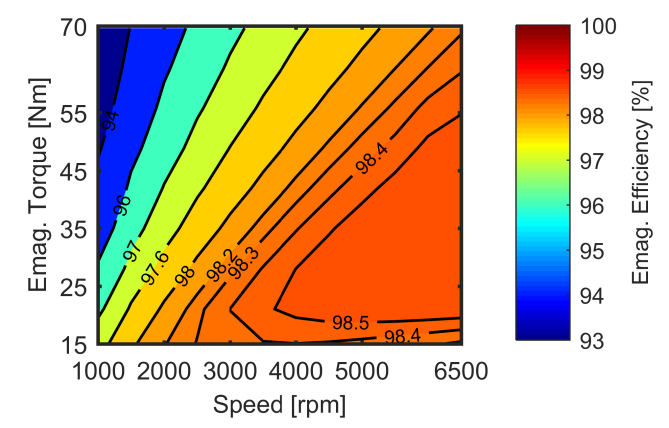

(b)

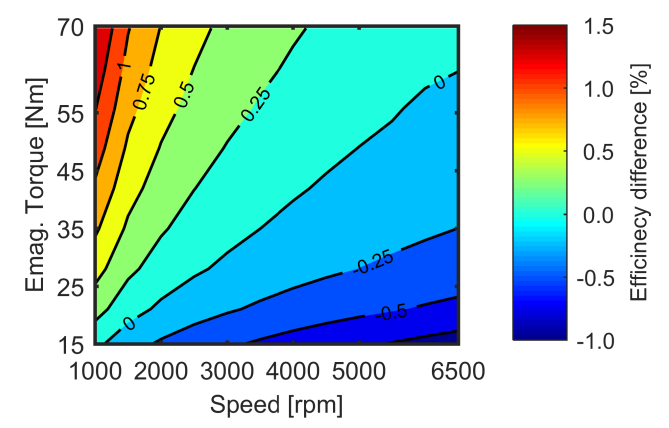

(c)

Fig. 11: The electromagnetic efficiency maps calculated by 3D FEA for the two representative designs with similar mass and loss: (a) single sided design, (b) the YASA design, and (c) the difference between efficiency maps of the two designs (the efficiency of the YASA machine subtracted from the single sided). temperature rises for the stators and rotors, respectively, the very high efficiency YASA designs are lighter than their single sided counterparts of comparable torque and loss performance. On the other hand, single sided machines may be preferable for applications in which lower mass is of the essence, at the inevitable expense of increased electromagnetic losses.

Within the entire design space considered, the optimally designed YASA machines require heavier copper windings and rotor cores, and lower stator core mass, as compared with the single-stator single-rotor machines. The YASA machines also require increasingly more magnet mass within the design space region with higher loss and lower mass. Lower core loss and higher copper loss are noted for the YASA optimal designs over the entire design space. The winding eddy current losses are comparable for the two machine configurations, while the YASA machines have higher magnet eddy current losses.

The studies conducted in this paper indicate that the preferred axial flux PM motor topology may depend on the torque and speed driving cycle requirements. The efficiency maps of two representative designs with comparable loss and mass show that, at the operating points with higher torque and lower speed, the single sided machine exhibits a higher efficiency, while for higher speed and lower torque, the YASA design is more efficient.

\section{ACKNOWLEDGMENT}

The support of Regal Beloit Corporation, ANSYS Inc., University of Kentucky, the L. Stanley Pigman endowment and the SPARK program is gratefully acknowledged.

\section{REFERENCES}

[1] P. D. Evans and J. F. Eastham, "Disc geometry homopolar synchronous machine," IEE Proceedings, vol. 127, no. 5, Sept 1980.

[2] E. Spooner and B. J. Chalmers, "Torus: A slotless, toroidal-stator, permanent-magnet generator," IEE Proceedings, vol. 139, no. 6, Nov 1992.

[3] T. J. Woolmer and M. D. McCulloch, "Analysis of the yokeless and segmented armature machine," in 2007 IEEE International Electric Machines Drives Conference, vol. 1, May 2007, pp. 704-708.

[4] B. Zhang, T. Seidler, R. Dierken, and M. Doppelbauer, "Development of a yokeless and segmented armature axial flux machine," IEEE Transactions on Industrial Electronics, vol. 63, no. 4, pp. 2062-2071, April 2016

[5] W. Fei, P. C. K. Luk, and K. Jinupun, "A new axial flux permanent magnet segmented-armature-torus machine for in-wheel direct drive applications," in 2008 IEEE Power Electronics Specialists Conference, June 2008, pp. 2197-2202.

[6] B. Zhang, T. Epskamp, M. Doppelbauer, and M. Gregor, "A comparison of the transverse, axial and radial flux pm synchronous motors for electric vehicle," in 2014 IEEE International Electric Vehicle Conference (IEVC), Dec 2014, pp. 1-6.

[7] N. J. Stannard, J. G. Washington, and G. J. Atkinson, "A comparison of axial field topologies employing smc for traction applications," in 2016 19th International Conference on Electrical Machines and Systems (ICEMS), Nov 2016, pp. 1-6.

[8] N. Taran, G. Heins, V. Rallabandi, D. Patterson, and D. M. Ionel, "Torque production capability of axial flux machines with single and double rotor configurations," in 2018 IEEE Energy Conversion Congress and Exposition (ECCE), Sep. 2018, pp. 7336-7341.

[9] N. Taran, D. M. Ionel, and D. G. Dorrell, "Two-level surrogate-assisted differential evolution multi-objective optimization of electric machines using 3-d fea," IEEE Transactions on Magnetics, vol. 54, no. 11, pp. 1-5, Nov 2018.

[10] C. E. Rasmussen and C. K. I. Williams, Gaussian Processes for Machine Learning. MIT Press, 2006. 